\title{
Analysis of peripapillary vessel density and Bruch's membrane opening-based neuroretinal rim parameters in glaucoma using OCT and OCT-angiography
}

\author{
Philip Enders $\mathbb{1}^{1}$ - Valentina Longo ${ }^{1,2}$ - Werner Adler ${ }^{3}$ Jens Horstmann ${ }^{1}$ - Friederike Schaub ${ }^{1}$ - Thomas Dietlein ${ }^{1}$. \\ Claus Cursiefen ${ }^{1} \cdot$ Ludwig M. Heindl $^{1}$
}

Received: 10 December 2018 / Revised: 14 August 2019 / Accepted: 25 September 2019 / Published online: 24 October 2019

(c) The Author(s), under exclusive licence to The Royal College of Ophthalmologists 2019

\begin{abstract}
Purpose To compare peripapillary vessel density (VD) measured by spectral domain optical coherence tomography angiography (SD-OCT-A) with morphometric parameters assessing the neuroretinal rim based on Bruch's membrane opening (BMO) by spectral domain optical coherence tomography (SD-OCT) in glaucoma.

Methods In this non-interventional cohort study, 50 eyes of 25 consecutively enrolled patients with diagnosis of glaucoma underwent SD-OCT and SD-OCT-A imaging of the optic nerve head (ONH). BMO minimum rim width (BMO-MRW) and area (BMO-MRA) as well as peripapillary retinal nerve fiber layer (RNFL) thickness were compared to peripapillary VD in the RNFL layer around the ONH.

Results Mean BMO-MRW was $221.46 \pm 81.5 \mu \mathrm{m}$, mean BMO-MRA was $1.05 \pm 0.04 \mathrm{~mm}^{2}$, mean RNFL thickness was $72.46 \pm 23.16 \mu \mathrm{m}$, and mean VD was $43.8 \pm 11.4 \%$. VD was significantly lower when morphometric parameters had lower thickness values $(p<0.01)$. Correlation coefficients and their 95\%- confidence intervals $(95 \%$-CI) with VD were $r=0.53$ (95\%-CI: 0.21-0.77) for BMO-MRW, $r=0.55$ (95\%-CI: 0.21-0.77) for BMO-MRA, and $r=0.57$ (95\%-CI: 0.13-0.73) for RNFL thickness. Intra-individual VD in both eyes correlated with $r=0.72(p<0.001)$, mean VD was comparable $(p=0.6)$. Eyes with high global RNFL thickness $(>90 \mu \mathrm{m})$ showed less VD variance $\left(\sigma^{2}=48.1\right)$ compared to eyes with highly reduced RNFL thickness $\left(<65 \mu \mathrm{m} ; \sigma^{2}=82.0\right)$. Best corrected visual acuity, perimetric mean defect, and PSD correlated significantly with VD (95\%-CI: -0.66 to $-0.10,0.16$ to 0.6 , and -0.65 to -0.02 , respectively).

Conclusions Peripapillary vessel density measured by SD-OCT angiography correlates significantly with Bruch's membrane opening-based parameters measured by SD-OCT in glaucoma patients.
\end{abstract}

\section{Introduction}

Optical coherence tomography (OCT) of the optic nerve head $(\mathrm{ONH})$ to measure and quantify neuroretinal tissue is now widely used in glaucoma diagnostics and follow-up $[1,2]$. As an extension of this technique, optical coherence

Philip Enders

philip.enders@uk-koeln.de

1 Department of Ophthamology, University of Cologne, Faculty of Medicine and University Hospital Cologne, Kerpener Strasse 62, 50924 Cologne, Germany

2 University of Applied Sciences Koblenz, Koblenz, Germany

3 Department of Biometry and Epidemiology, Friedrich-Alexander University Erlangen-Nuremberg, Erlangen, Germany tomography angiography (OCT-A) allows imaging of retinal and choroidal blood vessels [3-5]. Calculation of vascular density for different retinal layers (e.g., superficial and deep vascular plexus) as well as for the choroid is based on analysis of images obtained by OCT-A. For patients with glaucoma, numerous studies using OCT-A could demonstrate a reduction of vessel density (VD) and blood flow index within the $\mathrm{ONH}$, peripapillary retina and the macula [3, 6-18]. In a recent review on OCT-A imaging in glaucoma, the authors concluded that OCT-A has a high repeatability and reproducibility as well as good discriminatory power to differentiate normal from glaucoma eyes [19]. Furthermore, OCT-A is more strongly correlated with visual field function than conventional OCT and allows detection of glaucoma progression [19].

Different OCT algorithms and technical solutions offered by various manufacturers allow OCT-A imaging [19]. For 
glaucoma diagnostics, most of these studies on OCT-A used a comparison to peripapillary or macular parameters measuring neuroretinal tissue as peripapillary or macular retinal nerve fibre layer thickness ( $\mathrm{pRNFL}$ or mRNFL), total rim area or macular ganglion cell complex thickness [3, 20]. In addition to these parameters, morphometric parameters based on Bruch's membrane opening have been proposed [2, 21-23]. While Bruch's membrane opening-based minimal rim width (BMO-MRW) is available for clinical routine in some imaging solutions, the newer two dimensional parameter Bruch's membrane opening minimal rim area (BMO-MRA) is still under investigation in the preclinical stage [24-26].

In the present study, we prospectively recruited a cohort of patients with glaucoma that had been hospitalized within the clinical standard operating procedures to obtain a 24-hours intraocular pressure (IOP) profile including IOP measurements in supine position. The study aimed to characterize and compare peripapillary vascular density intra- and inter-individually to Bruch's membrane based morphometric parameters BMO-MRW, BMO- MRA as well as to circumpapillary RNFL thickness. Key focus was to assess the agreement of vascular density and Bruch's membrane opening based morphometry.

\section{Methods}

Data for this single-centre study were acquired from April to June 2018 at the Department of Ophthalmology, University Hospital of Cologne, Germany. After prospective enrolment, patients received SD-OCT and SDOCT-A measurements consecutively on the same day.

\section{Study subjects and inclusion criteria}

Inclusion criteria were hospitalization for 24-h IOP measurement due to presence of open angle glaucoma in clinical assessment. Patients with primary as well as open angle glaucoma due to pseudoexfoliation or pigment dispersion have been included. Written informed consent was obtained from all participants. Only examinations with an image quality index of $>15 \mathrm{~dB}$ were included in the analysis. Exclusion criteria were insufficiency of clear optical media defined by the ability to reach a sufficient SD-OCT quality index as defined, retinal or other pathology presenting any potential bias for imaging analysis (e.g., ONH drusen, peripapillary choroidal neovascularization, significant epiretinal membrane), inability for accurate fixation, recent intraocular surgery ( $<7$ days) or other causes for hospitalization than defined within the inclusion criteria. Participants' eyes were classified into three groups according to their peripapillary global RNFL thickness to reflect the degree of damage in neuroretinal tissue. Thresholds of RNFL thickness were set aiming at a balanced size of subgroups of the included eyes. High global RNFL thickness was defined as thickness of larger than $90 \mu \mathrm{m}$. Medium RNFL thickness was defined as ranging values between 65 and $89 \mu \mathrm{m}$, while low global RNFL thickness was $<65 \mu \mathrm{m}$. Additional information was obtained from patients' files. Standard automated perimetry (SAP) was performed using an Octopus 101 visual field analyser (Haag-Streit AG, Koeniz, Switzerland) with a 30:2 standard pattern with a TOP (threshold oriented perimetry) strategy. IOP was assessed by corneal rebound tonometry (Icare tonometer TA01i, Icare Finland Oy, Vantaa, Finland).

\section{SD-OCT imaging}

OCT examinations were performed by Spectralis $^{\circledR}$-SDOCT2 (Heidelberg Engineering GmbH, Heidelberg, Germany) according to standard operating and imaging procedures using a light source of $870 \mathrm{~nm}$. Included OCT data comprised an image quality index of $>15 \mathrm{~dB}$. The scanning pattern was centred on the BMO with radial equidistance (24-high resolution $15^{\circ}$ radial scans, each averaged from 27 B-scans). The examiner controlled the centration of the scan to the optic disc and corrected errors in detection of internal limiting membrane (ILM) and Bruch's membrane opening. All SD-OCT images were obtained by the same investigator (VL). BMO area, as well as Bruch's membrane opening minimum rim width (BMO-MRW) and retinal nerve fibre layer thickness were calculated by the device operating software tool provided by Heidelberg Engineering (software version 6.9.4.1), including a data export batch provided by the manufacturer. BMO-MRA calculation was performed by Heidelberg Engineering non-marketed scientific software Spectralis SP-X VWM. Gardiner and associates proposed the principle of BMO-MRA calculation [24].

\section{OCT-Angiography imaging}

OCT-A imaging was performed equally with the Spectralis $^{\oplus}$-SD-OCT2 using the angiography module. Technique of OCT-A image acquisition has been extensively described before [3]. A pattern sized scan of $3.0 \mathrm{~mm} \times 3.0$ $\mathrm{mm}$ applied manually and centred on the ONH'. A total of 512 B-scans were obtained per eye. Device operating software was used to produce an OCT-A image of the ONH and peripapillary region displaying vascular components. Within the operating software of the OCT-A device, manual segmentation of retinal layers was applied in all B-scans. Focus in this study was on the vessel density in the peripapillary RNFL layer. Segmentation only included structures of the inner limiting membrane (ILM) and the lower boundary of the RNFL layer. Contrast was set to 1:3 for all 

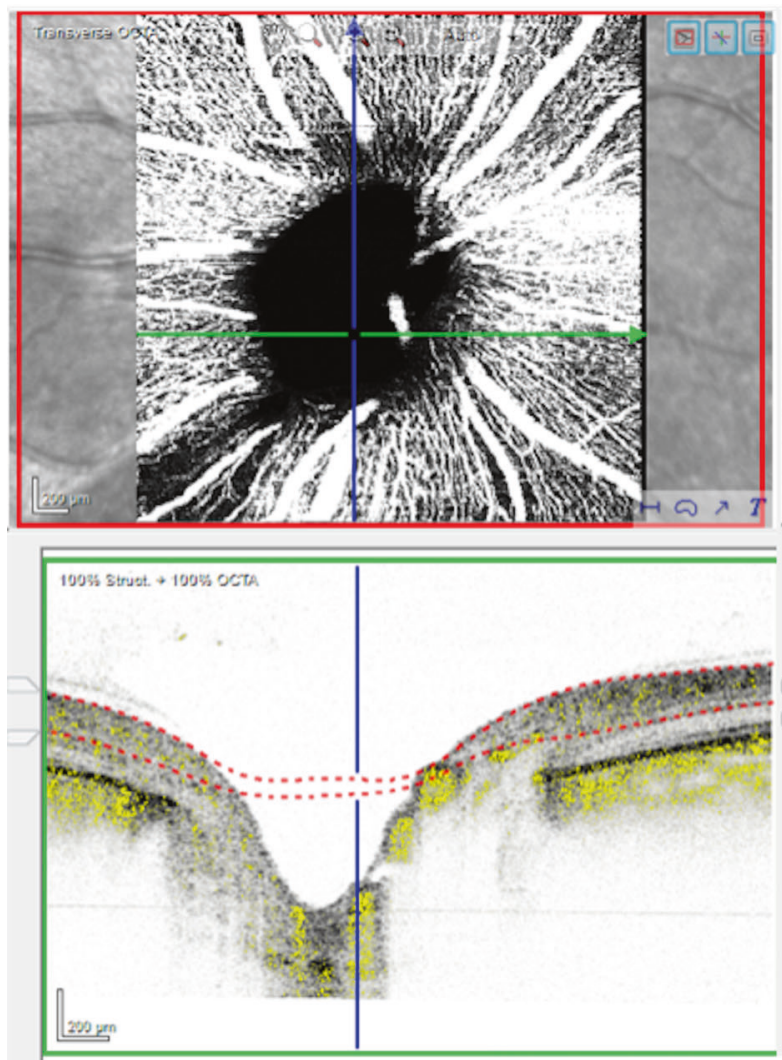

Fig. 1 Peripapillary vasculature in two glaucoma patients imaged by OCT-A. LEFT: Upper image displays peripapillary vasculature in the RNFL layer of the retina measured by OCT-A. The male patient was 84 years of age and had a perimetric mean defect of -17.4 decibel. Lower image illustrates exemplary B-scans showing OCT data (black), the OCT-A data as the vasculature (yellow) and the segmentation lines, which were corrected manually. RIGHT: Image displays peripapillary vasculature of the right eye of a female patient with mild

images. Figure 1 displays an OCT-A image after manual segmentation.

\section{Calculation of vessel density around the $\mathrm{ONH}$}

Images $3.0 \mathrm{~mm} \times 3.0 \mathrm{~mm}$ in size displaying vasculature around the $\mathrm{ONH}$ were extracted from the OCT-A device operating software. Further image processing was performed using ImageJ/Fiji (version May 2017), an open access Java-based image progressing software [27]. Image size was standardized for all images based on the $3 \times 3$ $\mathrm{mm}^{2}$ acquisition mode; to augment image sharpness, an unsharp mask filter was used; mask weight was set to 0.6 and radius to 10 pixels. Calculation of VD required binary imaging through a threshold strategy which has been described in several OCT-A studies [28-30]. Images were converted from 8-bit into a red, green, blue colour type. The red channel was selected as reference after splitting of the images into the red, green, and blue channels. A
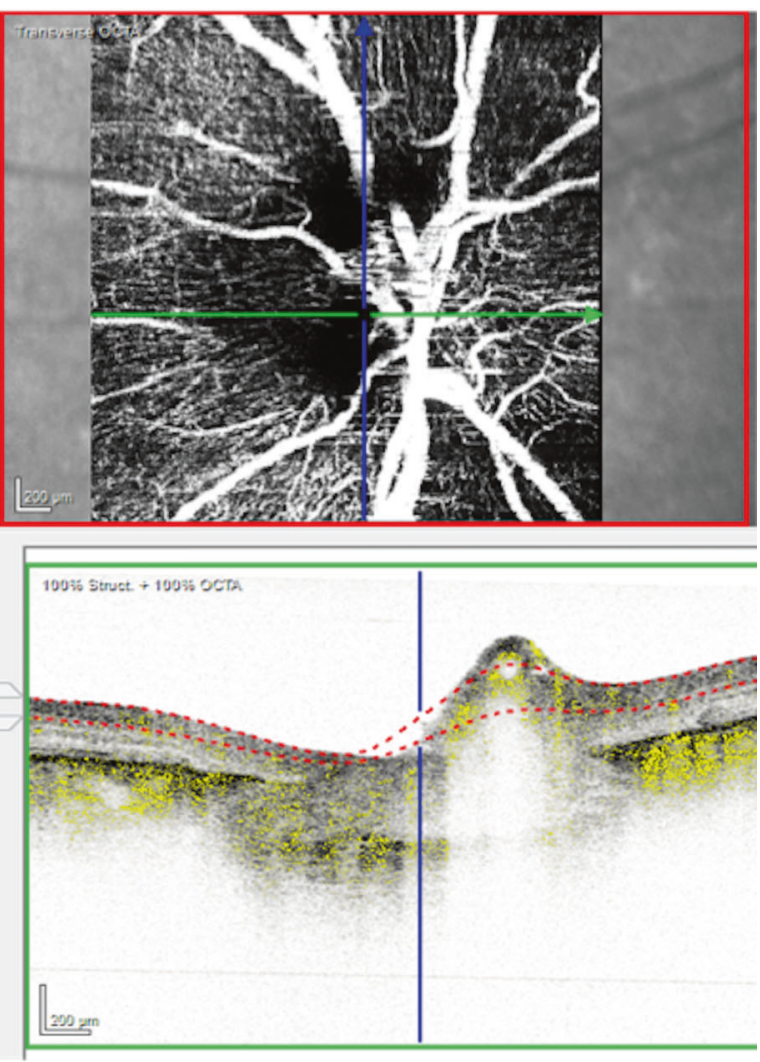

glaucomatous damage. The patient was 60 years of age at time of the examination. Perimetric mean defect was -3.3 decibel. Lower image illustrates exemplary B-scans showing OCT data (black), the OCT-A data as the vasculature (yellow) and the segmentation lines, which were corrected manually. Note: Respective Removal of big vessels and subtraction of BMO are not shown in these pictures. Legend: OCTA optical coherence tomography angiography, RNFL retinal nerve fiber layer; BMO Bruch's membrane opening

modified fixed threshold method was used [28]. All images were analysed with fixed thresholds at 60,65 , and 70 , VD results were averaged [31]. The fixed threshold method has been described to incorporate the best performance in distinguishing glaucomatous patients from healthy subjects [28]. In an additional step, larger vessels have been subtracted using a wand tracing tool. The individual BMO area was excluded in every image by marking the end of Bruch's membrane in all scans of an individual. This step was necessary to account for differences in BMO area size. Vessel density of retinal microvasculature around the $\mathrm{ONH}$ was calculated based on means of the proportion of bright pixels in the processed image. This semi-automated image processing procedure was repeated three times for every OCT-A image. The corresponding vessel density index corresponds closely to the parameter whole vessel density (wVD) described in studies using other imaging platforms. 


\section{Ethics and statistics}

Formal approval to conduct this study was obtained from the Ethics Committee (EC) of the University of Cologne (No. 16-340). Written informed consent has been obtained of all participants prior to additional SD-OCT and SD-OCTA examinations. All tenets of the declaration of Helsinki have been regarded. Statistical analyses were performed by SPSS (Version 24.0, IBM Corp. Armonk, NY, USA), R V 3.4.3 (R Foundation for Statistical Computing, Vienna, Austria. URL https://www.R-project.org/) and SAS 9.4 (SAS Institute Inc., Cary, NC, USA). To account for including two eyes per individual, mixed regression analyses were performed. Variables age, gender, IOP, and scan focus were provided as potential candidates for independent variables. The parameter(a) RNFL, (b) BMO-MRW, (c) BMO-MRA, and (d) visual mean defect, respectively was added as mandatory independent variable. After this, stepwise variable selection was performed to obtain four different mixed models with vessel density as dependent variable. Diagnostic plots did not raise any concerns regarding validity and appropriateness of these mixed models. The correlation between VD and the variables RNFL thickness, BMO-MRA, BMO-MRW, BCVA, MD, PSD, and IOP was estimated by mixed model regression using SAS 9.4 following the approach by Hamlett et al. which takes replication into account [32]. This approach also ensured that a strong correlation between both eyes of a patient was appropriately addressed within the statistical analysis.

\section{Results}

Thirty patients were enrolled in this study, whereof 50 eyes of 25 patients could be included in the following analyses. Ten eyes of five patients were excluded due to image acquisition errors or lacking image quality in OCT-A imaging. Epidemiology and baseline data of included patients are summarized in Table 1.

\section{Vessel density and morphometric parameters}

Mean VD in the RNFL layer around the ONH was $43.8 \pm$ $11.3 \%$ in all included eyes and ranged from $22.7 \%$ to $66.3 \%$. Mean VD was $44.3 \% \pm 11.1 \%$ in right and $43.2 \% \pm$ $11.8 \%$ in left eyes. Intra-individual VD in both eyes correlated with $r=0.72 \quad(p<0.001)$, while mean VD was comparable $(p=0.6)$. Mean BMO-MRW was $221.5 \pm 81.5$ $\mu \mathrm{m}$, mean BMO-MRA was $1.05 \pm 0.4 \mathrm{~mm}^{2}$, mean RNFL thickness was $73.0 \pm 22.2 \mu \mathrm{m}$ for all included eyes (Table 2). Stepwise selection of variables resulted in three mixed models including the independent variables age and
Table 1 Epidemiologic and baseline data

\begin{tabular}{|c|c|}
\hline \multicolumn{2}{|l|}{ Gender $n(\%)$} \\
\hline Men & $16(64.0)$ \\
\hline \multicolumn{2}{|l|}{ Eye $n(\%)$} \\
\hline Right & $25(50.0)$ \\
\hline \multicolumn{2}{|l|}{ Age (years) } \\
\hline Mean \pm SD & $60.8 \pm 21.1$ \\
\hline Median & 65.0 \\
\hline Range & 25 to 85 \\
\hline \multicolumn{2}{|c|}{ Current refractive error (scan focus, diopters) } \\
\hline Mean \pm SD & $-1.70 \pm 1.59$ \\
\hline Median & -1.43 \\
\hline Range & -5.12 to 0.92 \\
\hline \multicolumn{2}{|c|}{ Intraocular pressure (IOP, $\mathrm{mmHg}$ ) } \\
\hline Mean \pm SD & $16.8 \pm 5.3$ \\
\hline Median & 16.5 \\
\hline Range & 10 to 34 \\
\hline \multicolumn{2}{|c|}{ Best corrected visual acuity (BCVA, logMAR) } \\
\hline Mean \pm SD & $0.12 \pm 0.1$ \\
\hline Median & 0.1 \\
\hline Range & -0.1 to 0.5 \\
\hline \multicolumn{2}{|c|}{ Mean deviation (MD, decibel) } \\
\hline Mean \pm SD & $-8.1 \pm 7.1$ \\
\hline Median & -7.8 \\
\hline Range & -21.7 to 2.7 \\
\hline \multicolumn{2}{|c|}{ Pattern standard deviation (PSD, decibel) } \\
\hline Mean $\pm \mathrm{SD}$ & $5.0 \pm 2.8$ \\
\hline Median & 4.9 \\
\hline Range & 0.8 to 11.9 \\
\hline
\end{tabular}

$S D$ standard deviation, $B C V A$ best corrected visual acuity, IOP intraocular pressure, $d B$ decibel, $n / a$ not applicable, $B M O-M R W$ Bruch's membrane opening minimal rim width, BMO-MRA Bruch's membrane opening minimal rim area, $R N F L$ retinal nerve fiber layer thickness

IOP and the variables RNFL, BMO-MRW, and BMOMRA, that were defined as mandatory in the models (Table 3). VD was significantly lower when RNFL or BMO-MRW had lower thickness values (RNFL: $p=0.013$; BMO-MRW: $p=0.048)$. The association between VD and BMO-MRA was not significant $(p=0.127)$. The patients' age and IOP were found to have a statistically significant impact in all models $(p<0.007)$. Correlation coefficients that were calculated using mixed models to take into account presence of two eyes per subject between morphometric ONH parameters and VD were $r=0.53(95 \%$ bootstrap confidence intervals, 95\%-CI: $0.21,0.77$ ) for BMO-MRW, $r=0.55$ (95\%-CI: 0.21, 0.77) for BMO-MRA and $r=0.57$ (95\%-CI: 0.13, 0.73) for RNFL thickness. Bootstrap estimated 95\%-CI's for differences of correlation coefficients were $-0.34,0.19$ for the comparison of 
Table 2 Global and sector morphometric parameters of $\mathrm{ONH}$ analysis

\begin{tabular}{lccccccc}
\hline & Global & Nasal & Nasal superior & Nasal inferior & Temporal & Temporal superior & Temporal inferior \\
\hline Peripapillary Vessel density $(\%)$ & $43.76 \pm 11.34$ & - & - & - & - & - & - \\
BMO area $\left(\mathrm{mm}^{2}\right)$ & $2.13 \pm 0.56$ & - & - & - & - & - & - \\
BMO-MRW $(\mu \mathrm{m})$ & $221.46 \pm 81.46$ & $253.65 \pm 78.87$ & $245.19 \pm 85.35$ & $269.39 \pm 114.50$ & $166.10 \pm 70.19$ & $195.31 \pm 100.21$ & $211.10 \pm 107.66$ \\
BMO-MRA, $\left(\mathrm{mm}^{2}\right)$ & $1.05 \pm 0.44$ & $0.37 \pm 0.13$ & $0.13 \pm 0.05$ & $0.14 \pm 0.06$ & $0.20 \pm 0.10$ & $0.10 \pm 0.06$ & $0.12 \pm 0.06$ \\
RNFL thickness $(\mu \mathrm{m})$ & $73.02 \pm 22.24$ & $64.80 \pm 21.55$ & $80.92 \pm 28.59$ & $78.54 \pm 28.41$ & $59.72 \pm 18.14$ & $89.52 \pm 35.59$ & $95.00 \pm 44.65$ \\
\hline
\end{tabular}

Table present mean values $\pm \mathrm{SD}$

$S D$ standard deviation, $B M O-M R W$ Bruch's membrane opening minimal rim width, BMO-MRA Bruch's membrane opening minimal rim area, $R N F L$ retinal nerve fiber layer thickness

Table 3 Mixed regression models to assess impact of covariates on vascular density of the optic nerve head

\begin{tabular}{|c|c|c|c|}
\hline & Coefficient & $95 \%$ CI & $P$ Value \\
\hline \multicolumn{4}{|c|}{ Model 1 (Age, IOP, RNFL thickness) } \\
\hline (Intercept) & 65.325 & 50.39 to 80.259 & $<0.001$ \\
\hline Age & -0.285 & -0.428 to -0.142 & 0.001 \\
\hline IOP & -0.749 & -1.124 to -0.375 & $<0.001$ \\
\hline Global mean RNFL thickness & 0.114 & 0.03 to 0.198 & 0.013 \\
\hline \multicolumn{4}{|l|}{ Model 2 (Age, IOP, BMO-MRW) } \\
\hline (Intercept) & 65.406 & 47.94 to 82.872 & $<0.001$ \\
\hline Age & -0.271 & -0.432 to -0.11 & 0.002 \\
\hline IOP & -0.723 & -1.117 to -0.329 & 0.001 \\
\hline Global mean BMO-MRW & 0.031 & 0.001 to 0.061 & 0.048 \\
\hline \multicolumn{4}{|l|}{ Model 3 (Age, IOP, BMO-MRA) } \\
\hline (Intercept) & 67.145 & 48.033 to 86.258 & $<0.001$ \\
\hline Age & -0.277 & -0.449 to -0.104 & 0.003 \\
\hline IOP & -0.724 & -1.134 to -0.315 & 0.001 \\
\hline Global mean BMO-MRA & 0.005 & -0.001 to 0.012 & 0.127 \\
\hline \multicolumn{4}{|l|}{ Model 4 (Age, IOP, mean defect) } \\
\hline (Intercept) & 72.311 & 58.993 to 85.629 & $<0.001$ \\
\hline Age & -0.265 & -0.433 to -0.096 & 0.005 \\
\hline IOP & -0.594 & -0.994 to -0.194 & 0.006 \\
\hline Mean defect & 0.377 & 0.078 to 0.676 & 0.018 \\
\hline
\end{tabular}

$B M O-M R W$ Bruch's membrane opening minimal rim width, BMO-MRA Bruch's membrane opening minimal rim area, $R N F L$ retinal nerve fiber layer thickness, IOP intraocular pressure, $C I$ confidence interval
RNFLthickness/VD and BMO-MRA/VD, $-0.33,0.21$ for comparison of RNFL thickness/VD and BMO-MRW/VD, and $-0.08,0.11$ for comparison of BMO-MRW/VD and BMO-MRA/VD. Figure 2 shows the relationship between morphometric parameters RNFL, BMO-MRA, BMO-MRW and MD and Vascular Density.

Eyes with high global RNFL thickness $>90 \mu \mathrm{m}$ showed less VD variance $\left(\sigma^{2}=48.1\right)$ compared to eyes with highly reduced RNFL thickness of $<65 \mu \mathrm{m}\left(\sigma^{2}=82.0\right)$.

\section{Vessel density and functional parameters/IOP}

Best corrected visual acuity correlated significantly with $\mathrm{VD}, r$ was -0.42 (95\%-CI: -0.66 to -0.10$)$. In perimetric testing, twenty-one eyes had a MD better than $-6 \mathrm{~dB}$, while 14 eyes had a MD between $-6 \mathrm{~dB}$ and $>-12 \mathrm{bB}$. Eyes with a MD better than $-6 \mathrm{~dB}$ had a mean VD of $49.15 \pm 10.7 \%$.
Eyes with an MD ranging from -6 to $-12 \mathrm{~dB}$ had a mean VD of $40.29 \pm 10.4 \%$. Eyes with severe functional damage worse than $-12 \mathrm{~dB}$ had a mean VD of $35.7 \pm 8.0 \%$. The fourth mixed model in Table 3 shows that additionally to age and IOP, MD also had a significant influence, where higher MD resulted in higher VD $(p=0.018)$.

MD correlated significantly with VD, $r$ was 0.49 with a 95\%-CI between 0.16 and 0.69. Correlation for PSD was $r=-0.40$ (95\%CI: -0.65 to -0.02 ). IOP at examination did not correlate significantly with VD $(r=-0.30 ; 95 \%$ CI: -0.53 to 0.05 ).

\section{Discussion}

The results of this study confirm a significant association between vessel density around the $\mathrm{ONH}$ measured by 

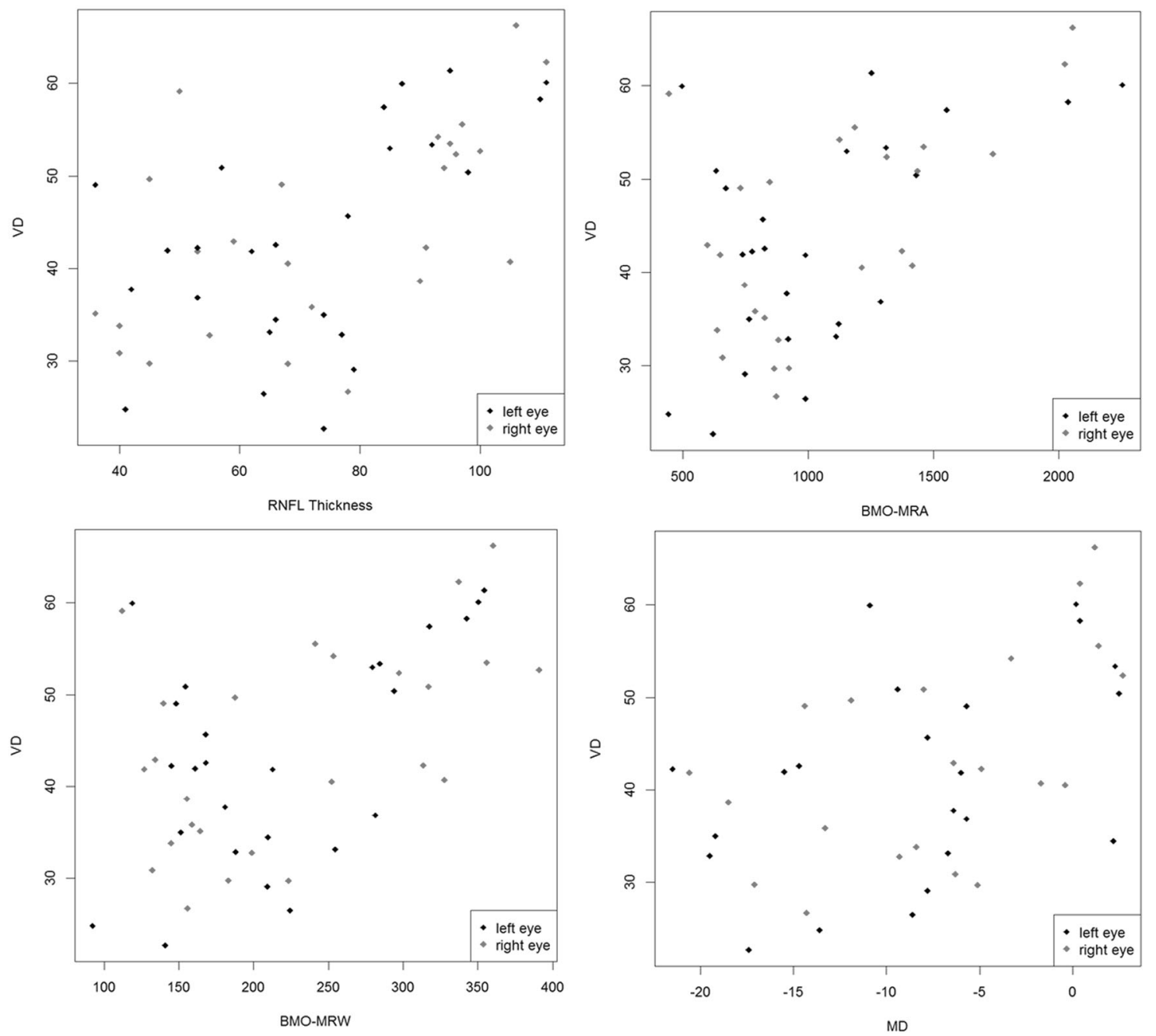

Fig. 2 : Scatter plots of VD compared to morphometric SD-OCT and functional parameters. Legend: VD, vascular density; SD-OCT, spectral domain optical coherence tomography; RNFL, retinal nerve

SD-OCT-A and Bruch's membrane opening-based parameters BMO-MRW and BMO-MRA measured by SD-OCT. The degree of association is comparable to conventional morphometric parameters as RNFL thickness. This finding is somewhat surprising as OCT-A imaging in our study assessed retinal microvasculature in the retinal nerve fibre layer. While BMO-based parameters have not been assessed so far, Yarmohammadi and associates reported weaker associations of total rim area and whole image VD (Pearson's $r=0.65$ ) compared to circumpapillary RNFL thickness and whole image VD (Pearson's $r=$ 0.83 ) using a different imaging platform [5]. With progression to severe glaucomatous damage, circumpapillary RNFL thickness incorporates a flooring effect [1]. In consequence, further worsening of glaucoma cannot be detected morphometrically once the threshold for flooring is reached [33]. Quantification of BMO-MRW and BMOMRA takes place at an anatomical location where more

fiber layer; BMO-MRA, Bruch's membrane opening minimal rim area; BMO-MRW, Bruch's membrane opening minimal rim width; $\mathrm{MD}$, mean defect

neuroretinal tissue per scan is present compared to the cirumpapillary retina [34]. Reason for this is an increase of neuroretinal tissue volume towards the optic nerve head. A shift in this flooring effect towards higher losses of neuroretinal tissue may be hypothesized for BMO-MRW and BMO-MRA. This could be leading to an increased discriminatory power towards an association of BMO-based parameters and VD in the presence of severe glaucoma.

Numerous studies on OCT-A for glaucoma demonstrated a link between the density of retinal microvasculature around the $\mathrm{ONH}$ and glaucomatous visual field loss $[1,19,33]$. Our study could show a correlation coefficient of $r=0.49$ for $\mathrm{VD}$ and MD. The mixed regression model confirmed the significant relationship between MD and VD. In consequence, our study can contribute in showing the significant interdependency between visual field loss and decrease vascular density. Yarmohammadi et al. reported a correlation of $r=0.71$ for the equivalent comparison [5]. 
Due to significant variations in imaging algorithms, platforms and approaches in OCT-A studies, comparability of results is somewhat limited [19]. Furthermore, it is crucial to take into account which retinal layers are under investigation to quantify vessel density. This study focused on vascular density in the RNFL layer. The approach aimed to reduce potential projection artefacts and should facilitate segmentation of retinal layers in the $\mathrm{ONH}$ area [3]. Other studies analysed vessel density in the whole retina between internal limiting membrane and Bruch's membrane opening $[3,19]$. Aging is known to result in a physiological loss in morphometric parameters BMO-MRW, BMO-MRA and RNFL thickness. The mixed regression models showed an impact of aging on vascular density of the $\mathrm{ONH}$ also in our study group. In many patients glaucoma severity is closely linked to aging, as structure and function deteriorates over the course of the disease. In consequence, normative VD data on healthy subject in different age groups will be helpful to better understand the impact of aging.

Limitations of our study are a drop-out of $17 \%$ of enrolled patients due to insufficient image quality or problem in image acquisition due to extensive acquisition time. Further standardization of VD calculation by an automated image processing tool is desirable to increase comparability between studies. Differences in refractive error could incorporate a bias on image area and pixel sizes. Significance of this potential scaling bias needs to be better understood. Image artefacts occurring in late stage RNFL loss might impact VD measurements as well. To improve understanding of the correlation and the variation of VD compared to morphometric SD-OCT parameters, analysis of larger patient cohorts seems desirable.

In summary, we could show that peripapillary VD measured by SD-OCT angiography correlates significantly with Bruch's membrane opening-based parameters measured by SD-OCT. No statistical difference between correlations for BMO-MRA and BMO-MRW with VD could be shown when compared to the correlation between peripapillary RNFL thickness and VD. The clinical implications of quantifying VD in glaucoma needs to be further evaluated.

\section{Summary}

\section{What was known before}

- Optical coherence tomography angiography (OCT-A) is investigated as additional diagnostic tool in glaucoma. Several studies could show correlations of vascular density measured by OCT-A and peripapillary retinal nerve fibre layer thickness (RNFL).

\section{What this study adds}

- In addition to the peripapillary RNFL thickness, other morphometric parameters based on Bruch's membrane opening (BMO) have been introduced to measure neuroretinal tissue. This study contributes by first describing the comparison of BMO-based parameters to OCT-A in glaucoma.

Acknowledgements We thank all technical experts of our imaging laboratory and well as FOR 2240 "(Lymph-) Angiogenesis and Cellular Immunity In Inflammatory Diseases Of The Eye" for their support.

\section{Compliance with ethical standards}

Conflict of interest The authors declare that they have no conflict of interest.

Publisher's note Springer Nature remains neutral with regard to jurisdictional claims in published maps and institutional affiliations.

\section{References}

1. Phillips DL, Hager JL, Goins KM, Kitzmann AS, Greiner MA, Cohen AW, et al. Boston type 1 keratoprosthesis for chemical and thermal injury. Cornea. 2014;33:905-9.

2. Chauhan BC, O'Leary N, Almobarak FA, Reis AS, Yang H, Sharpe GP, et al. Enhanced detection of open-angle glaucoma with an anatomically accurate optical coherence tomographyderived neuroretinal rim parameter. Ophthalmology. 2013;120: 535-43.

3. Svedbergh B, Jonsson V, Afzelius B. Immotile-cilia syndrome and the cilia of the eye. Albrecht Von Graefes Arch Klin Exp Ophthalmol. 1981;215:265-72.

4. Svedbergh B. Effects of artificial intraocular pressure elevation on the outflow facility and the ultrastructure of the chamber angle in the vervet monkey (Cercopithecus ethiops). Acta Ophthalmol. 1974;52:829-46.

5. Yarmohammadi A, Zangwill LM, Diniz-Filho A, Suh MH, Yousefi S, Saunders LJ, et al. Relationship between optical coherence tomography angiography vessel density and severity of visual field loss in glaucoma. Ophthalmology. 2016;123:2498-508. Epub 2016/10/12

6. Krypton laser photocoagulation for idiopathic neovascular lesions. Results of a randomized clinical trial. Macular Photocoagulation Study Group. Arch Ophthalmol. 1990;108:832-7.

7. Evens RA, Bardsley B, CM VK. Auditory complaints in scuba divers: an overview. Indian $\mathbf{J}$ Otolaryngol Head Neck Surg. 2012;64:71-8

8. SC CM, Carapuca HM, Duarte AC. Ion-exchange and permselectivity properties of poly(sodium 4-styrenesulfonate) coatings on glassy carbon: application in the modification of mercury film electrodes for the direct voltammetric analysis of trace metals in estuarine waters. Talanta. 2005;65:644-53.

9. Lu JF, Barron-Casella E, Deering R, Heinzer AK, Moser AB, deMesy Bentley KL, et al. The role of peroxisomal ABC transporters in the mouse adrenal gland: the loss of Abcd2 (ALDR), 
Not Abcd1 (ALD), causes oxidative damage. Lab Invest. 2007;87:261-72.

10. Svedbergh B, Sherwood M. Argon laser trabeculoplasty in exfoliation glaucoma. A retrospective analysis. Dev Ophthalmol. 1985;11:116-23.

11. Svedbergh B, Backlund Y, Hok B, Rosengren L. The IOP-IOL. A probe into the eye. Acta Ophthalmol. 1992;70:266-8.

12. Sherwood MB, Svedbergh B. Argon laser trabeculoplasty in exfoliation syndrome. Br J Ophthalmol. 1985;69:886-90.

13. Svedbergh B. Argon laser trabeculoplasty in capsular glaucoma. Acta Ophthalmol Suppl. 1988;184:141-7.

14. Svedbergh B, Alm A, Amer B, Jerndal T, Thorburn W. Goniodysgenesis in elderly glaucoma and non-glaucoma patients. A masked slit-lamp and gonioscopy study. Acta Ophthalmol. 1986;64:45-51.

15. Buchwald HJ, Weingartner WE, Spraul CW, Wagner P, Lang GK. Contusion of the optic nerve after minor blunt ocular trauma: case report and literature review. Ophthalmologe. 1999;96:529-33. Contusio nervi optici nach geringgradigem stumpfen Bulbustrauma. Ein Fallbericht mit Literaturubersicht

16. Meier P, Wiedemann P. Vitreoretinal surgery in children. Ophthalmologe. 1999;96:536-54. Vitreoretinale Chirurgie bei Kindern

17. Toth G, Bucher F, Siebelmann S, Bachmann B, Hermann M, Szentmary N, et al. In situ corneal cross-linking for recurrent corneal melting after boston type 1 keratoprosthesis. Cornea. 2016;35:884-7.

18. Raghavan U, Downes R, Jones NS. Spontaneous resolution of eyeball displacement caused by maxillary sinusitis. $\mathrm{Br} \mathrm{J}$ Ophthalmol. 2001;85:118.

19. Bill A, Svedbergh B. Scanning electron microscopic studies of the trabecular meshwork and the canal of Schlemm-an attempt to localize the main resistance to outflow of aqueous humor in man. Acta Ophthalmol. 1972;50:295-320.

20. Horn FK, Nguyen NX, Mardin CY, Junemann AG. Combined use of frequency doubling perimetry and polarimetric measurements of retinal nerve fiber layer in glaucoma detection. Am J Ophthalmol. 2003;135:160-8.

21. Sit AJ, Liu JH. Pathophysiology of glaucoma and continuous measurements of intraocular pressure. Mol Cell Biomech. 2009;6:57-69.

22. Todani A, Behlau I, Fava MA, Cade F, Cherfan DG, Zakka FR, et al. Intraocular pressure measurement by radio wave telemetry. Invest Ophthalmol Vis Sci. 2011;52:9573-80.
23. Lin CC, Chen A, Jeng BH, Porco TC, Ou Y, Han Y. Scleral intraocular pressure measurement in cadaver eyes pre- and postkeratoprosthesis implantation. Invest Ophthalmol Vis Sci. 2014;55:2244-50.

24. Gardiner SK, Ren R, Yang H, Fortune B, Burgoyne CF, Demirel $\mathrm{S}$. A method to estimate the amount of neuroretinal rim tissue in glaucoma: comparison with current methods for measuring rim area. Am J Ophthalmol. 2014;157:540-9 e1-2.

25. Yung E, Trubnik V, Katz LJ. An overview of home tonometry and telemetry for intraocular pressure monitoring in humans. Graefes Arch Clin Exp Ophthalmol. 2014;252:1179-88.

26. Wachtlin J, Gamulescu MA. Age-related macular degeneration. Klin Monbl Augenheilkd. 2012;229:941-55. quiz56-7. Altersabhangige Makuladegeneration

27. Algvere P, Jonsson V, Svedbergh B. Vascular damage in the anterior uvea induced by intravitreal autogenous blood. Albrecht Von Graefes Arch Klin Exp Ophthalmol. 1981;217:273-83.

28. Rabiolo A, Gelormini F, Sacconi R, Cicinelli MV, Triolo G, Bettin $\mathrm{P}$, et al. Comparison of methods to quantify macular and peripapillary vessel density in optical coherence tomography angiography. PLoS ONE. 2018;13:e0205773.

29. Cicinelli MV, Rabiolo A, Marchese A, de Vitis L, Carnevali A, Querques L, et al. Choroid morphometric analysis in nonneovascular age-related macular degeneration by means of optical coherence tomography angiography. $\mathrm{Br} \mathrm{J}$ Ophthalmol. 2017;101:1193-200. Epub 2017/01/07

30. Battaglia Parodi M, Rabiolo A, Cicinelli MV, Iacono P, Romano F, Bandello F. Quantitative analysis of optical coherence tomography angiography in adult-onset foveomacular vitelliform dystrophy. Retina. 2018;38:237-44.

31. Yen JC, Chang FJ, Chang S. A new criterion for automatic multilevel thresholding. IEEE Trans Image Process. 1995;4:370-8. Epub 1995/01/01

32. Hamlett A, Ryan L, Serrano-Trespalacios P, Wolfinger R. Mixed models for assessing correlation in the presence of replication. $\mathrm{J}$ Air Waste Manag Assoc 2003;53:442-50.

33. Svedbergh B. Retrolental fibroplasia or congenital encephaloophthalmic dysplasia? Acta Paediatr Scand. 1975;64:891-4.

34. Danthurebandara VM, Sharpe GP, Hutchison DM, Denniss J, Nicolela MT, McKendrick AM, et al. Enhanced structure-function relationship in glaucoma with an anatomically and geometrically accurate neuroretinal rim measurement. Invest Ophthalmol Vis Sci. 2015;56:98-105. 